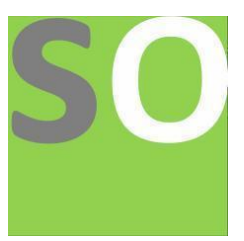

Article title: Mixed Integer Programming Technique According to Traffic Light for Autonomous Collision Free Ground Based on Traffic System

Authors: Nahida Islam[1], Dr. Mohammad Hanif Hanif[2]

Affiliations: Applied Mathematics, Noakhali Science and Technology University, Noakhali-3814[1], Professor of Applied Mathematics, Noakhali Science and Technology University, Noakhali-3814[2]

Orcid ids: 0000-0002-8402-5787[1]

Contact e-mail: mail.nahidaislam@gmail.com

License information: This work has been published open access under Creative Commons Attribution License http://creativecommons.org/licenses/by/4.0/, which permits unrestricted use, distribution, and reproduction in any medium, provided the original work is properly cited. Conditions, terms of use and publishing policy can be found at https://www.scienceopen.com/.

Preprint statement: This article is a preprint and has not been peer-reviewed, under consideration and submitted to ScienceOpen Preprints for open peer review.

DOI: 10.14293/S2199-1006.1.SOR-.PPJ4WKZ.v1

Preprint first posted online: 01 September 2021

Keywords: Mixed Integer Programming 


\section{Mixed Integer Programming Technique According to Traffic Light for Autonomous Collision Free Ground Based on Traffic System}

\author{
Nahida Islam \\ Noakhali Science and \\ Technology University, \\ Noakhali-3814, \\ Bangladesh \\ mail.nahidaislam@gmail.c \\ om
}

\author{
Dr. Mohammad Hanif \\ Professor, \\ Noakhali Science and \\ Technology University, \\ Noakhali-3814, \\ Bangladesh \\ drhanifmurad@yahoo.co
}

\begin{abstract}
The optimization process has been applied in the present study for a collision-free self-directed ground-Based traffic system. An algorithm has been proposed, called MIPATS (Mixed integer programming Algorithm for self-directed ground-based Traffic system), which makes the highperformance crossway crossing discrete-time trajectory for every transport. The Constraints are very well designed and the process has been optimized to make collisions free crossway crossing system. The potency of the suggested MIPATS is measured through a protracted model using a microscopically dealings simulator, ANYLOGIC. The pretending results show that MIPATS performance is better traffic throughput compared to a general traffic light model.
\end{abstract}

\section{General Terms}

2010 Mathematics Subject Classification, Mathematical programming 9OCxx, Mixed integer programming 90C11

\section{Keywords}

Connected self-directed transport, Ground-Based traffic System, Level-headed Crossway Direction, Linear Programming, Mixed Integer Programming, Road traffic network, Transport to Crossway communication.

\section{INTRODUCTION}

Considering a possible outcome to the traffic over-crowded trouble, self-directed conveyance systems, especially selfdirected crossway control, have attracted considerable research and development measures. To stop more than twoway crossing the solution to the problem in this field will be how to make better traffic protection and throughput by using traffic lights or stop signs and holding the advantages of self-

$\mathrm{m}$

directed transports. Researchers all over the world have discussed about various optimization strategies to enrich the throughput which dynamically develops a sequence of collision-free motions among those a nonlinear programming formulation with relaxing nonlinear constraints a model predictive control framework [2], Each self-directed transport minimizes energy consumption subject to the requirements of throughput and protection which is a decentralized framework [3], etc are some of them. Most of these moments are at the equivalent time trying to avoid the presence of a transport with an opposing pathway inside a crossway to ensure protection. Allowing conflicting vehicles exist inside a crossway concurrently some researchers studied algorithms to further enrich performance. Most strategies discredited the crossway space into grid cells to achieve this objective so that vehicles of the conflicting pathway can exist at the equivalent time within a crossway, but not within the equivalent cell. In [4], the Self-directed Crossway Direction was suggested which allows route-conflicting vehicles to enter the crossway concurrently as long as they don't occupy the equivalent cell at the equivalent time. However, to obtain optimal traffic flow no global adjustment is made to cross the transport. There is a lot of research work focused on the optimization of selfdirected crossway control, and a lot of effort is being spent to allow route-conflicting vehicles to appear within the equivalent crossway to enrich efficiency. However, allowing the concurrent presence of conflicting vehicles within a crossway several researchers have tried to optimize the crossway control. Dai et al. [5] transformed the crossway control model into a convex optimization problem with an objective function of multiple criteria like safe speeds and accelerations to make better the quality of experience from passenger's perspective. They have linearized to avoid collisions by setting the precedence for transport access at crossways to alleviate the computational cost. However for example; this linearization can reduce the effectiveness; For a schedule, the train arriving earlier requires the first collision to cross the collision zone for two collided vehicles. Reference [6] suggested a vehicle-crossway coordination scheme that develops smooth flows of traffic by preventing any pair of conflicting vehicles from approaching their Cross-Collision Point at the equivalent time. Considering the state of all vehicles, the paper developed a constrained nonlinear optimization Problem to reduce the risk function to generate 
safe trajectories of vehicles with unused time and space in the crossway space. On the other hand, the algorithm needs more computation costs to coordinate more vehicles since they optimize the traffic flow of all approaching vehicles at the equivalent time. And the optimization problem is nonlinear, thus the solution is not sure to be the global optimum. This paper proposes an optimization formulation similar to [5], [6] for crossway control, but comes through their limitations in terms of fixed scheduling and nonlinearity of problem formulation based on level-headed crossway coordination algorithms [7]-[9]. We have several major contributions as follows. Our formulation operates with only one transport (see Chapter 2 for definition), which reduces the computation load of the central control unit and the Communication needs among vehicles, the crossway, and the traffic light. The algorithm optimizes the trajectory of a new head transport using Mixed Integer Programming (MIP) under various constraints thus called MIPATS (Mixed integer programming Algorithm for self-directed ground-based Traffic system). The main contribution of the paper is the modeling of potential collisions with space-conflicting vehicles using mixed-integer programming according to traffic lights, introducing variables that represent the potential behaviors of a transport. As a result of this modeling, MIPATS allows a later arrived transport exits the crossway before the earlier conflicting transport, depending on the length of cross-area, probability of throughput, speed, and acceleration if performance will be increased. We also modeled immediate onwards vehicles and road cross pathways in the crossway area as constraints in the developed problem to ensure the transport is free from collisions. The problem formulation is simple and achieves the global optimal trajectory for a transport. The simulation results show that MIPATS reduces average trip time and fairness among vehicles significantly over existing methods. The remainder of this paper is organized into five chapters. Chapter 2 introduces the basic assumptions and interaction scheme between a crossway and a transport for MIPATS. The trajectory optimization problem is developed in Chapter 3 with each constraint explained detailed. In Chapter 4, we propose the MIPATS algorithm incorporating the optimization approach. The Efficiency of MIPATS is evaluated through extensive simulations in Chapter 5. Finally, Chapter 6 concludes the paper.

\subsection{Aim and objectives}

Within the optimized traffic system

- To arrange collision-free traffic.

- Providing a safe road traffic system for the V2I communication system.

- To minimize road traffic time and increase the performance of the traffic system.

- Used properly traffic light system by the advantage of self-directed vehicles.

- The probability of traffic flow is considered in the suggested traffic light system.

- Initial speed, volume, acceleration, declaration are properly calculated and controlled, for this why it performs substantially better.

\section{TRANSPORT-CROSSWAY INTERACTION}

In the present study we survey in the crossway, we suppose that all vehicles are connected as well as self-directed. So we name them CAVs where each CAV can wirelessly communicate Vehicle-to-Crossway (V2I), and also can avoid collision with its immediate onwards transport when imminent a crossway. We also assume that all CAVs are able to obtain their speeds, positions, and relative distances to an intersecting entrance line (see Figure 2). In the present study, crossway traffic is controlled in a central way by the crossway controller, called the Crossway Control Agent (ICA). As mentioned above, the ICA of a crossway communicates with CAVs wirelessly via V2I communication. We define a region, called the contact zone, around a crossway in which CAV can communicate with ICA wirelessly. It is also assumed that each CAV is equipped with an RFID chip. Through the detectors installed at the entrance of the communication region, ICA can detect each CAV's VIN (Vehicle's Identification Number), approaching lane, and also the time when the transport enters the communication region.

Figure 1 shows the high-level interaction between a CAV and ICA. In the crossway control framework suggested in the present study, we say that a CAV becomes a head transport only when there are no other vehicles between the CAV and the entrance of the crossway, or the transport which is immediately in onwards of the CAV has begun to enter the crossway. If a transport becomes a head transport, it

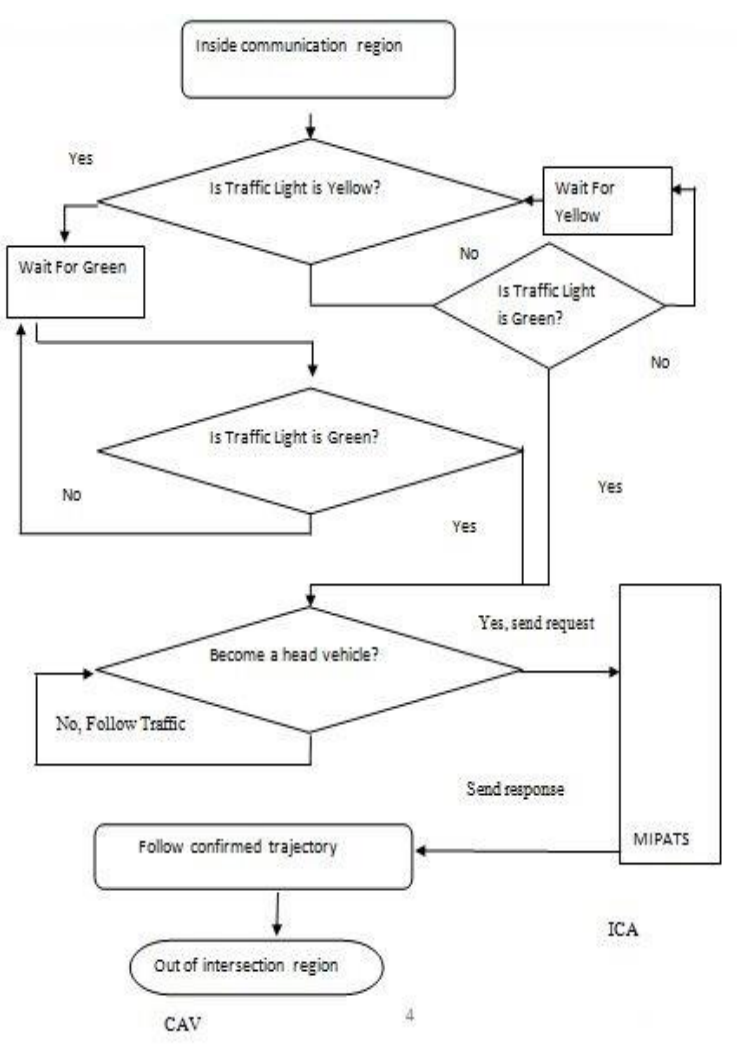

Fig 1: Intersection between $\mathrm{CAV}$ and ICA

Immediately sends a REQUEST message to the ICA asking to generate a crossing trajectory for any transport to follow. We 
call such a trajectory a guaranteed trajectory. We suppose that a REQUEST message contains all the necessary information, such as a vehicle's current speed, position, crossway crossing path, etc. to generate a confirmed trajectory of the ICA. After a confirmed trajectory is generated, the ICA sends the trajectory to the transport as a RESPONSE message. We call a transport that has received a confirmed trajectory a confirmed transport. If transport is not a major transport, it will continue to drive other transports to follow automatically. Note that the ICA can determine if the transport is a major transport or not because such information can easily be collected from the detectors described above. Therefore, REQUEST messages sent accidentally from non-head vehicles are ignored only by ICA.

\section{PROBLEM FORMULATION}

In this section, we address the general traffic system and refer to the problem as a statement .After we describe our new formulation to make better the overall crossway crossing traffic throughput. Through a mixed integer programming formulation, we resolve the problem of trajectory generation of each transport for crossway crossing which ensures that vehicles can cross an crossway as soon as possible while collisions among vehicles are safely avoided.

\subsection{The General Traffic system:}

A traffic system where traffic control is not optimized, fixed traffic light duration is set up for traffic control, Critical traffic flow duration is not considered, the road is not well-formed design such as median strip width, intersecting pathway are not well-formed designed. This kind of traffic system is called the general traffic system.

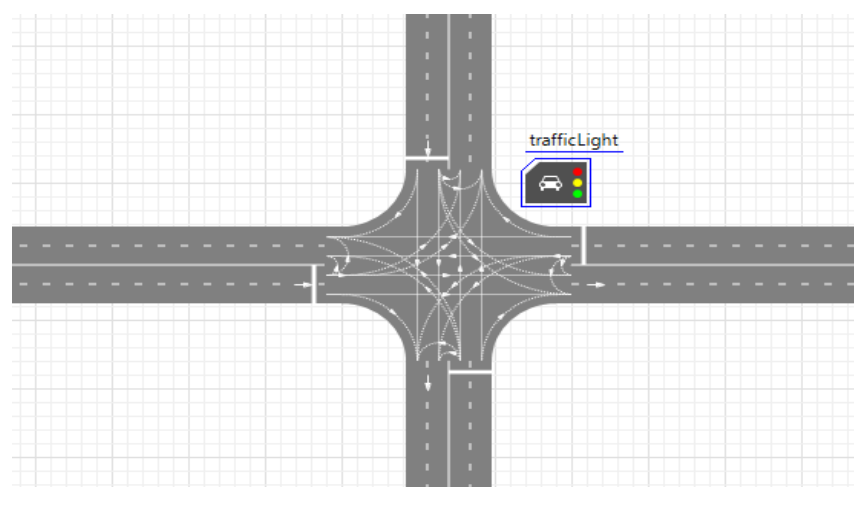

Fig 2: Road are not well-formed design. Median strips are not counted correctly.

And throughput probability is not considered in general traffic systems.

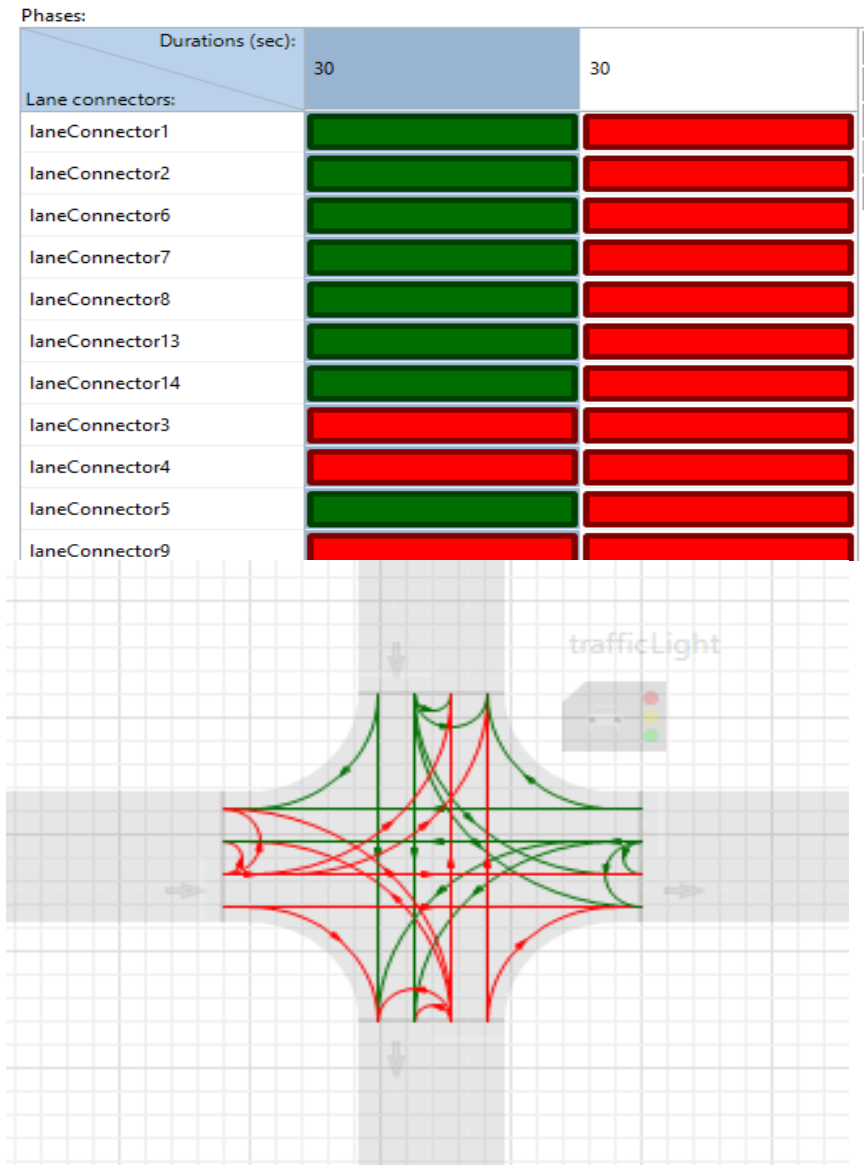

Fig3: Critical traffic flow duration is not considered in general traffic system.

\subsection{Statement of the problem:}

In general traffic system:

When the Traffic light period is fixed for the traffic light, the traffic flow will not be according to the density, speed, acceleration, and deceleration of the transport. To increase the traffic jam, it reduces the protection of the traffic network. The traffic congestion will increase and the efficiency of the traffic system will decrease.

When Roads are not well-formed designed and critical traffic flow time has not been set up so that collisions occur in the crossway area.

It maximizes the meantime of road traffic network means car starting time and end time of a traffic network.

Initial speed, volume, acceleration, declaration are not properly calculated, for this why nearly collisions will be increased.

The probability of traffic flow is not considered in the general traffic system. 


\subsection{Single Transport Trajectory Formation}

Considering the trajectory generation for a single-transport we first started to create a mixed integer programming optimization problem. In this case, we suppose that there is only one transport near the crossway. Then, our object in this simple situation is to develop a discrete-time trajectory for the transport so that it can cross the crossway as quickly as possible while satisfying various constraints such as speed, traffic light and acceleration limits.

When transport crosses a crossway, let $\gamma$ be the pathway (or path) for the transport to obey and also let $\mathrm{s}$ be the variable that represents the position of the transport along the path. We suppose that $\gamma$ is fixed for the given incoming and outgoing lanes across a crossway. We also use $\mathrm{h}$ to denote the fixed sampling time, $\mathrm{v}_{\text {max }}$ to denote the maximum speed limit for the transport, $\mathrm{a}_{\max }$ and $\mathrm{a}_{\min }$ to denote the maximum and minimum acceleration rates respectively. Note that, for a fixed $\mathrm{h}$, we can represent the discrete-time trajectory of a transport as a sequence of positions of the transport along $\gamma$ such as $\left(s_{0}, s_{1}, s_{2}, \ldots, s_{k}, \ldots, s_{n}\right)$ for some positive integer number $n$ where $s_{\mathrm{k}}$ represents the position of the transport along at the $\mathrm{k}$ th time step. Here, we implicitly suppose that $s_{i} s_{j}$ if $i<j$ and this relation will be incorporated as a constraint in our optimization problem formulation below. Choose $\mathrm{n}$ as a sufficiently large number. Let $s=\left[s_{0} s_{1} \ldots s_{n}\right]^{T} \in \mathbb{R}^{n}$. Then the fastest crossing discrete-time trajectory for a transport can be obtained by

$\max$

$s \in \mathbb{R}^{n} S_{n}$

under some constraints which are described below.

The first constraint that we should consider is the order relationship between any pair of $s_{i}$ and $s_{j}$ in $s \in \mathbb{R}^{n}$ where $i, j$ $\in[0 ; \mathrm{n}]$. Since $\mathrm{s}$ should represent an ordered sequence of positions of the transport along $\gamma$ at each sample time step, it is clear that we should constraint the values for each variable $\mathrm{s}_{\mathrm{k}}$ in $\mathrm{s}$ as follows:

$\mathrm{s}_{\mathrm{k}}^{\mathrm{lb}} \leq \mathrm{s}_{\mathrm{k}} \leq \mathrm{s}_{\mathrm{k}}^{\mathrm{ub}}$

Where,

$$
\begin{gathered}
s_{\mathrm{k}}^{\mathrm{lb}}=0 \text { if } \mathrm{k}=0 ; \mathrm{s}_{\mathrm{k}}^{\mathrm{lb}}=\mathrm{s}_{\mathrm{k}-1} \text { if } \mathrm{k} \neq 0 ; \\
\mathrm{s}_{\mathrm{k}}^{\mathrm{ub}}=\mathrm{s}_{\mathrm{k}+1} \text { if } \mathrm{k} \neq 0 ; \mathrm{s}_{\mathrm{k}}^{\mathrm{ub}}=\mathrm{v}_{\max } \cdot \mathrm{n} \cdot \mathrm{h} \text { if } \mathrm{k}=\mathrm{n}
\end{gathered}
$$

Subsequently, we note that vehicles should not be driven backward on the roads and should obey the law enforcement speed limit. Thus, we have the following constraint for speed:

$0 \leq \mathrm{v}_{\mathrm{k}} \leq \mathrm{v}_{\max }$

Where $\mathrm{v}_{\mathrm{k}}$ is the speed of the transport at the $\mathrm{k}$ th time step. In order to incorporate this constraint into our optimization formulation in (1), we need to rewrite the velocity variable $v_{k}$ in terms of the positions $s_{k}$ in $s$ as

$\mathrm{v}_{\mathrm{k}}=\frac{\mathrm{s}_{\mathrm{k}}-s_{\mathrm{k}-1}}{\mathrm{~h}}$ for $\mathrm{k} \in[1, \mathrm{n}]$
In addition to the speed constraint, we also consider constraints for the acceleration (or deceleration) rate of a transport in order to make sure the discrete-time trajectory generated by the optimization process is dynamically feasible. Let $a_{k}$ be the acceleration rate of a transport at the kth time step. Then, the constraint for acceleration $\mathrm{a}_{\mathrm{k}}$ is given by

$\mathrm{a}_{\min } \leq \mathrm{a}_{\mathrm{k}} \leq \mathrm{a}_{\max }$ for $\mathrm{k} \in[1, \mathrm{n}]$

Again, in order to incorporate these constraints into the optimization formulation in (1), it is necessary to represent $a_{k}$ in terms of the variables $s_{k}$ in $s$ as follows:

$\mathrm{a}_{1}=\frac{\mathrm{v}_{1}-\mathrm{v}_{\mathrm{o}}}{\mathrm{h}}=\frac{\mathrm{s}_{1}-\mathrm{s}_{0}-\mathrm{v}_{\mathrm{o}} \mathrm{h}}{\mathrm{h}^{2}}$

$\mathrm{a}_{\mathrm{k}}=\frac{\mathrm{v}_{\mathrm{k}}-\mathrm{v}_{\mathrm{k}-1}}{\mathrm{~h}}=\frac{\mathrm{s}_{\mathrm{k}}-2 \mathrm{~s}_{\mathrm{k}-1}+\mathrm{s}_{\mathrm{k}-2}}{\mathrm{~h}}$ for $\mathrm{k} \in[2, \mathrm{n}]$

Where $\mathrm{v}_{\mathrm{o}}$ is the speed of the transport at the 0th time step which we suppose is known to ICA via direct measurement or V2I message sent from the transport to ICA at that moment.

\subsection{Mixed Integer Programming for Collision Avoidance:}

In this section, we generalize the best discrete-time trajectory generation problem to include situations when other vehicles also formulation an crossway. In this situation, due to the existence of other vehicles, we need to develop additional constraints and include them in the optimization process so that the transport of interest can

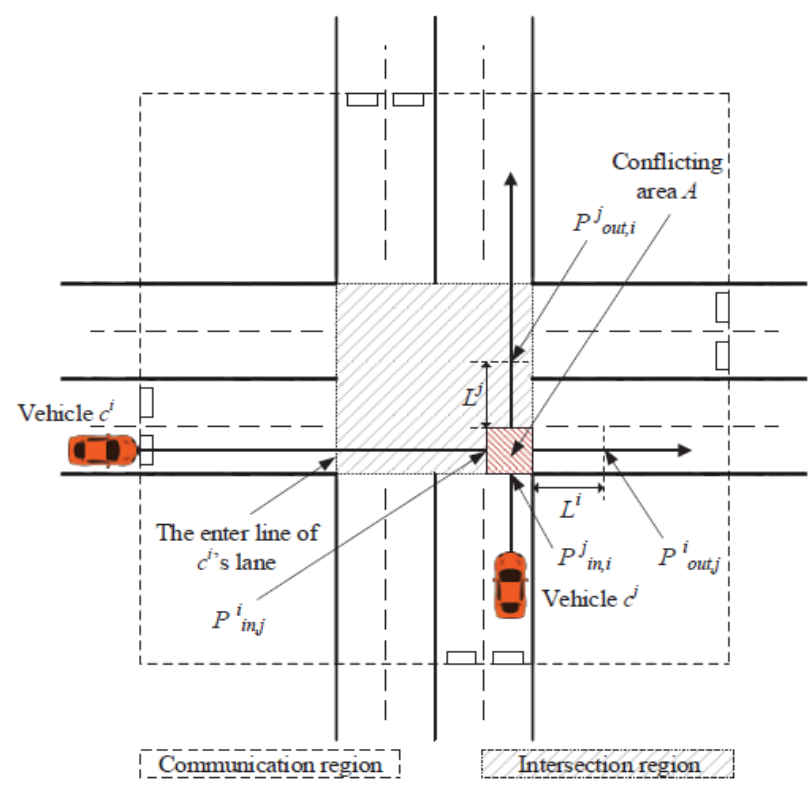

Fig 4: Examples of conflicting area.

Successfully cross a crossway while avoiding collisions with other vehicles. In our discussion below, we can use the 
superscript $i$ to indicate the transport of interest for which the best discrete-time trajectory is currently being developed.

\subsubsection{Constraints from space-conflicting vehicles:}

Suppose that there is a set of vehicles $\mathrm{C}$ which has reached the communication area rather than the transport $c^{i}$ and so their optimal discrete-time trajectories to cross the crossway have already been generated and confirmed. Let $\mathrm{C}^{*}$ be the set of vehicles in $\mathrm{C}$ whose pathway are conflicting with the transport $c^{i}$ s pathway $\gamma^{i}$. Now, let us consider a transport $c^{j} \in$ $\mathrm{c}^{*}$ to derive collision avoidance constraints for $\mathrm{c}^{\mathrm{i}, \mathrm{s}}$ optimal trajectory generation. For this, we note first that, as discussed in our paper [8], both ends of an intersecting path (entering and exiting of an crossway) are separate from each other, whereas space conflicts (or overlapping) occur only on their pathway . Also, one can see that these space conflicting short ranges along the pathway can be set offline for all pairs of conflicting crossway crossing pathway . Thus, to make the transport $c^{\mathrm{i}}$ avoid any potential collisions with vehiclec ${ }^{j}$, it is necessary to ensure that $c^{i}$ does not occupy the space conflicting area when $c^{j}$ occupies that area.

Figure 4 illustrates the situation for example when the transport $c^{j}$ is already a confirmed transport as the vehiclec $c^{i}$ is entering the communication region. In this situation, since there is no other transport in onwards of $c^{\mathrm{i}}$ in its lane, $c^{\mathrm{i}}$ is the head transport and being considered for confirmation by ICA (i.e., the optimal discrete-time trajectory is being generated by ICA). The two long lines with arrows represent the pathway of the two vehicles. Their pathway has conflicting area A (with red diagonal lines), which is the area where both vehicles should not be occupied at the equivalent time to avoid collisions. In the figure, $p_{i n, j}^{i}$ denotes the location on $\gamma^{\mathrm{i}}$ that $c^{\mathrm{i}}$ starts to enter the area $\mathrm{A}$ and $\mathrm{p}_{\text {out,j }}^{\mathrm{i}}$ denotes the location on $\gamma^{\mathrm{i}}$ that $c^{\mathrm{i}}$ becomes free from collision with $c^{j}$.Note that we represent the location of a transport by the vehicle's onwards bumper location along its pathway. Thus we choose the location of $p_{\text {out,j }}^{\mathrm{i}}$ to be at one transport length $\left(\mathrm{L}^{\mathrm{i}}\right)$ away from the right boundary of A since the length of a transport should also be considered for collision avoidance. Note also that the locations $p_{i n, j}^{i}$ and $p_{\text {out }, j}^{i}$ along $\gamma^{i}$ are assumed to be known since they can be computed offline for every pair of crossway crossing pathway and, in the sequel, we use $\left.\gamma^{\mathrm{i}}\right|_{\mathrm{j}}$ to denote the conflicting area on $\gamma^{\mathrm{i}}$.

Now we note that ,for $c^{i}$,there are two ways to avoid any potential collisions withc ${ }^{\mathrm{j}}$ : (i) Arrive at $\left.\gamma^{\mathrm{i}}\right|_{\mathbf{j}}$ after $c^{\mathrm{j}}$ exits its $\left.\gamma^{j}\right|_{i}$, or (ii) exit $\left.\gamma^{i}\right|_{j}$ before $c^{j}$ arrives at its $\left.\gamma^{j}\right|_{i}$. To capture this logical condition when solving the optimization problem for trajectory generation for $c^{\mathrm{i}}$, we adopt a binary variable $b_{j}^{\mathrm{i}} \in$ $\{0,1\}$ to represent $c^{\mathrm{i},}$ s behavior with respect to $\mathrm{c}^{\mathrm{j}}$ and reformulate our optimization problem in (1) as a mixedinteger linear programming (MILP) problem. Let $p_{i n, j}^{i}$ and $\mathrm{p}_{\text {out,j }}^{\mathrm{i}}$ the distance from the transport $\mathrm{c}^{\mathrm{i}, \mathrm{s}}$ current position to $\mathrm{p}_{\mathrm{in}, \mathrm{j}}^{\mathrm{i}}$ and $\mathrm{p}_{\text {out }, \mathrm{j}}^{\mathrm{i}}$ along $\gamma^{\mathrm{i}}$ respectively. Then the constraints for collision avoidance with $c^{j}$ can be represented as

$$
s_{\tau(i n, j)}^{i} \geq p_{o u t, j}^{i}+M \cdot\left(b_{j}^{i}-1\right)
$$

$s_{\tau(\text { out }, j)}^{\mathrm{i}} \geq \mathrm{p}_{\mathrm{in,j}}^{\mathrm{i}}+\mathrm{M} \cdot \mathrm{b}_{\mathrm{j}}^{\mathrm{i}}$

Where $\mathrm{M}$ is an arbitrarily large number, $\mathrm{s}_{\mathrm{k}}^{\mathrm{i}}$ is the $\mathrm{c}^{\mathrm{i}, \mathrm{s}}$ position on $\gamma^{\mathrm{i}}$ at the $\mathrm{k}$ th time step, $\tau(\mathrm{in}, \mathrm{j}) \in\{0,1, \ldots \ldots, \mathrm{n}\}$ is the time step when $c^{j}$ completely leaves $\left.\gamma^{j}\right|_{i}$ i.e. $c^{j}$ arrives at $p_{\text {out,i }}^{j}$. Note that $\tau(\mathrm{in}, \mathrm{j})$ and $\tau$ (out, j) can be easily computed based on $\mathrm{c}^{\mathrm{j}}, \mathrm{s}$ confirmed optimal discrete-time trajectory. If $b_{j}^{i}=1,(7)$ holds and this means that $c^{i}$,is already out of the conflicting area at the time when $c^{j}$ enters the conflicting area. Similarly, if $b_{j}^{i}=0,(8)$ holds meaning that $c^{i}$ is still before the conflicting area at the time when $c^{j}$ exits the conflicting area. Thus, the binary variable $b_{j}^{i}$ ensures that the two equations cannot hold at the equivalent time.

Now we have modeled the constraints from one conflicting transport at $c^{*}$. Following the equivalent process, we can easily add all other vehicles to $c^{*}$ by computing the corresponding times and location parameters related to their conflicting areas with transport $c^{i}$.

\subsubsection{Constraints from onwards vehicles:}

The onwards transport crosses the crossway area according to the rules of figure 5 describe. The generated pathway pattern for vehicles is to take the left, through, U-Turn or right. Besides vehicles in $\mathrm{c}^{*}$ considered above, a transport that is immediately in onwards of $c^{i}$ may also have a direct influence on $\mathrm{c}^{\mathrm{i}}, \mathrm{s}$ crossing behavior since $\mathrm{c}^{\mathrm{i}}$ should plan its trajectory to avoid rear-end collision with its onwards transport. As shown in Figure 4, in crossing an crossway, $c^{\mathrm{i}}$ needs to avoid rearend collisions with three different types of onwards vehicles: (i) a onwards transport with the equivalent entrance lane $\left(c_{e n}^{f}\right)$, (ii) a onwards transport with the equivalent exit lane ( $\mathrm{c}_{\mathrm{ex}}^{\mathrm{f}}$ ), and (iii) a onwards transport with the equivalent pathway $\left(c_{r 0}^{f}\right)$. Below, we address how to constrain the speed of a transport $c^{i}$ to avoid these different types of collisions in onwards vehicles.

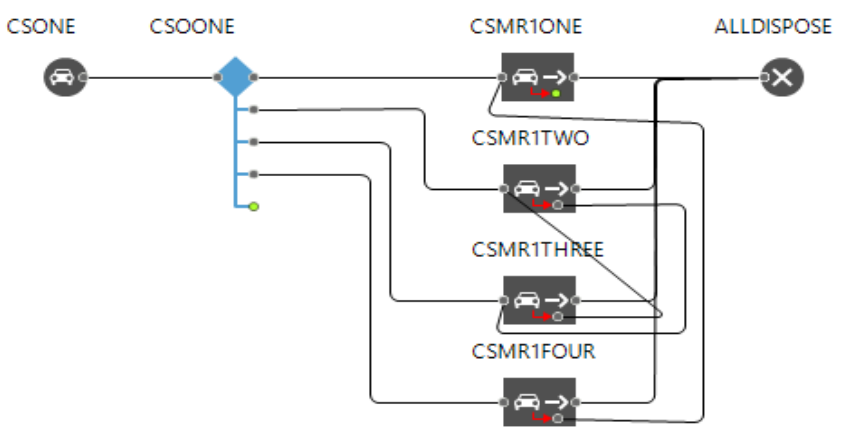



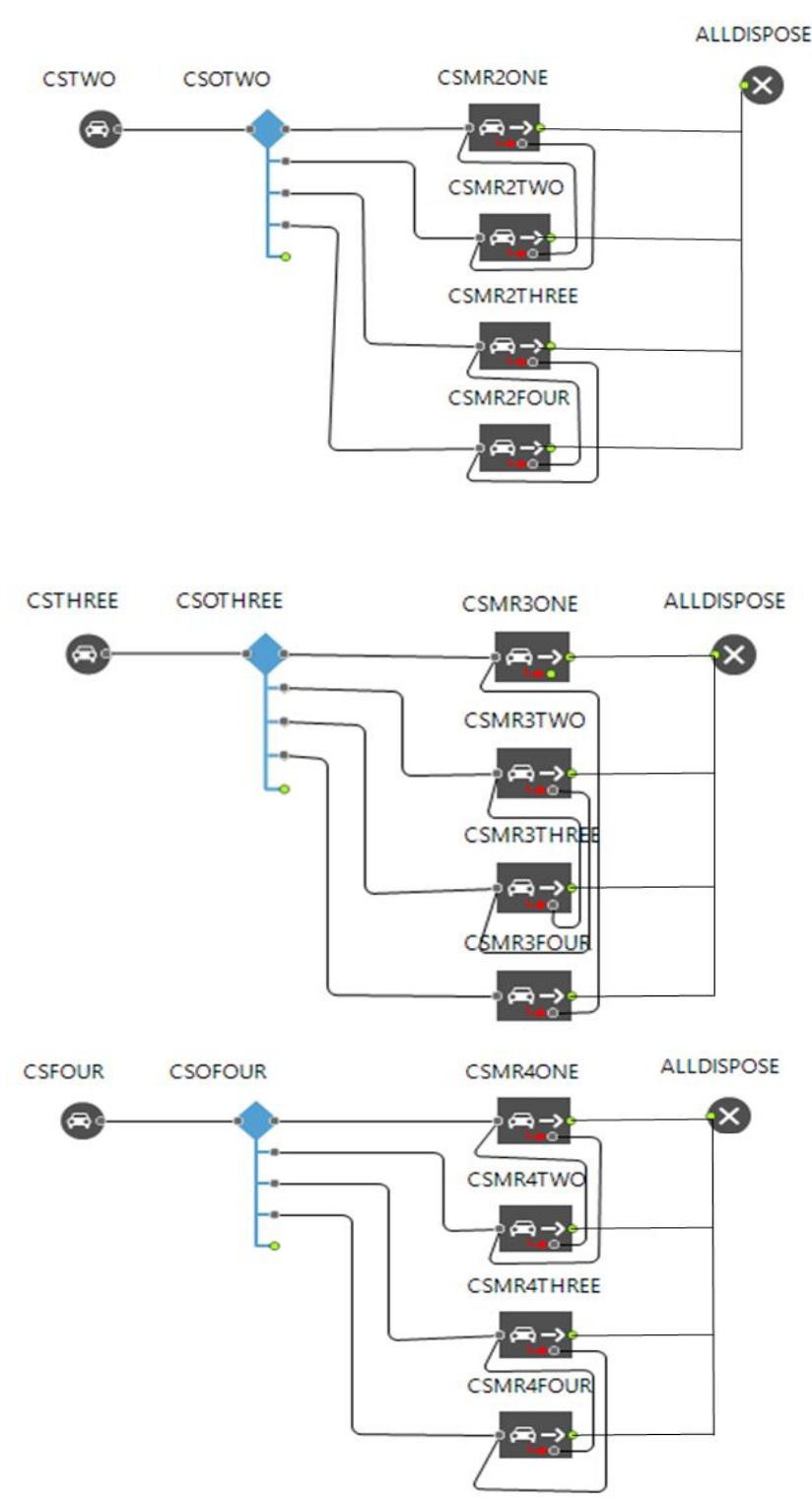

Fig 5: Communication and pathway process from each road to each road within crossway area.

In the following discussion, we also suppose that the onwards vehicles have already been confirmed by the ICA and therefore their crossway crossing trajectories are already known to the ICA.

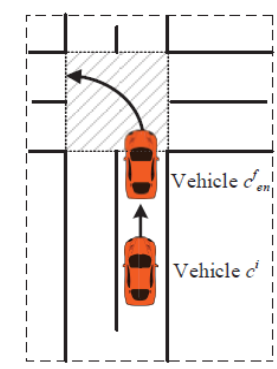

(a)

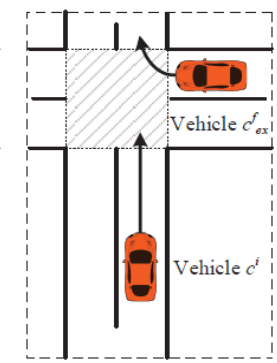

(b)

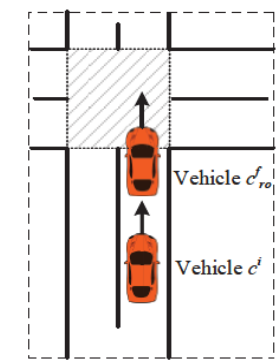

(c)
Fig 6: Examples of three types of onwards vehicles.
First, let us look at avoiding collisions with onwards transport $c_{e n}^{f}$. If we use $\tau(e n, f)$ to denote the time step when $c_{e n}^{f}$ enters the line of crossway and $v_{\tau(e n, f)}^{f}$ is the speed of $c_{e n}^{f}$ at that moment, then, to ensure that the transport $c^{i}$ does not collide with the $c_{e n}^{f}$ when $c^{i}$ is approaching the crossway, we constrain the speed of $c^{i}$ so that both vehicles are separated in time at least $t_{\text {sep }}$ which can be determined conservatively by assuming that $c^{i} s$ currently reaching an crossway at its maximum speed $v_{\max }$. Thus we define $t_{\text {sep }}$ as

$t_{\text {sep }}=\frac{\mathrm{v}_{\max }-\mathrm{v}_{\tau(\mathrm{en}, \mathrm{f})}^{\mathrm{f}}}{\left|\mathrm{a}_{\min }\right|}+\frac{\mathrm{g}_{\mathrm{min}}}{\mathrm{v}_{0}}+\delta$

In (9), $g_{\min }$ is a constant that represents the minimum distance interval between two adjacent vehicles on the equivalent lane regardless of their speeds and $\delta$ is another constant that represents a protection margin over time. The first term in (9) is the time $c^{i}$ needs to decelerate from $v_{\max }$ to $v_{\tau(e n, f)}^{f}$

With its maximum deceleration rate while the second one is the minimum time required to ensure the minimum distance interval $g_{\min }$. then we can use the following constraint to ensure that $c^{i}$ arrives at the crossway entrance after $\tau(e n, f)$ with a certain amount of time interval:

$\mathrm{s}_{\tau(\mathrm{en}, \mathrm{i})}^{\mathrm{i}} \leq \mathrm{p}_{\mathrm{en}}^{\mathrm{i}}$

Where $\tau($ en, $\mathrm{i})=\tau(\mathrm{en}, \mathrm{f})+\left[\frac{\mathrm{t}_{\mathrm{sep}}}{\mathrm{h}}\right]$ is the earliest time step at which $c^{i}$ can arrive at the entrance of the crossway, and $p_{e n}^{i}$ is the location of the crossway entrance on $\gamma^{\mathrm{i}}$.

Next, in order to avoid collision with the onwards vehicle $c_{\mathrm{ex}}^{\mathrm{f}}$, we can construct a constraint using the equivalent idea that introduces a minimum separation time $t_{\text {sep }}$ between $c^{i}$ and $c_{e x}^{f}$ when they depart an crossway. Specifically, we make $c^{i}$ exit the crossway at least $t_{\text {sep }}$ time later after $c_{e x}^{f}$ exits. In this case, $t_{\text {sep }}$ can be obtained in the equivalent way as in (9) except that $v_{\tau(e n, f)}^{f}$ must be replaced by $v_{\tau(e x, f)}^{f}$ in the first term of the equation where $\tau(\mathrm{ex}, \mathrm{f})$ is the time step when $c_{\mathrm{ex}}^{\mathrm{f}}$ exits the crossway and $v_{\tau(e x, f)}^{f}$ is the speed of $c_{e x}^{f}$ at that moment. Following a similar process, we can have the following constraint for $\mathrm{c}^{\mathrm{i}}$ to avoid collision with $\mathrm{c}_{\mathrm{ex}}^{\mathrm{f}}$ :

$\mathrm{s}_{\tau(\mathrm{ex}, \mathrm{i})}^{\mathrm{i}} \leq \mathrm{p}_{\mathrm{ex}}^{\mathrm{i}}$

Where $\tau(e x, i)=\tau(e x, f)+\left\lceil\frac{t_{\text {sep }}}{h}\right\rceil$ is the earliest time step at which $c^{i}$ can arrive at the entrance of the crossway and $p_{e x}^{i}$ is the location of the crossway entrance on $\gamma^{\mathrm{i}}$.

Now, to avoid collision with onwards vehicles $c_{e x}^{\mathrm{f}}$, we note that this can be done by combining the constraints between the first two types of onwards vehicles. Thus, we include both (10) and (11) at the equivalent time as a constraint to our optimization problem to constrain the speed of the transport $c^{i}$ to avoid such collisions of onwards vehicles. 


\subsection{Optimization Problem Formulation}

We now present the final form of the suggested optimization process to generate a discrete-time crossway crossing trajectory for the transport $\mathrm{c}^{\mathrm{i}}$ which avoids collisions with all confirmed vehicles. Collecting variables for optimization from (1), (7), and (8), we first define

$\mathrm{X}:=\left\{\mathrm{s}_{1}^{\mathrm{i}}, \mathrm{s}_{2}^{\mathrm{i}}, \ldots, \mathrm{s}_{\mathrm{n}}^{\mathrm{i}}, \mathrm{b}_{1}^{\mathrm{i}}, \mathrm{b}_{2}^{\mathrm{i}}, \ldots . ., \mathrm{b}_{1}^{\mathrm{i}}\right\}$ assuming that $\left|\mathrm{C}^{*}\right|=1$ and $x(k)$ indicates the $k$ th element of $x$. Then the final form of the optimization problem is to find $\mathrm{x}^{*}$ such that

$\mathrm{X}^{*}=\underset{\mathrm{x} \in \mathrm{R}^{\mathrm{n}} \times\{0,1\}^{1}}{\arg \max } \mathrm{x}(\mathrm{n})$

subject to constraints

Eqs. (2), (3), and (5).

For transport $C^{j} \in C^{*}$, eqs. (7) and (8).

For $c_{\mathrm{en}}^{\mathrm{f}}$ or $\mathrm{c}_{\mathrm{r} 0}^{\mathrm{f}}$ type onwards transport, eq. (10)

For $\mathrm{c}_{\mathrm{ex}}^{\mathrm{f}}$ or $\mathrm{c}_{\mathrm{r} 0}^{\mathrm{f}}$ type onwards transport, eq. (11)

Where $v_{k}^{i}$ and $a_{k}^{i}$ is defined as in (4) and (6), respectively.

\section{MIPBased Crossway Control}

In this chapter, we present the overall crossway control framework based on the optimization process introduced in Chapter 3. We call it the Mixed integer programming Algorithm for an self-directed ground-based Traffic system (MIPATS). As shown in Algorithm 1, the function is BestSolutionFeasible () is first called and returns constraints due to onwards vehicles. Then, the algorithm calls the function getBestIteration () to determine constraints due to vehicles whose confirmed trajectories are space conflicting with the pathway of $c^{i}$. After all necessary constraints for collision avoidance are obtained, the algorithm develops the optimal discrete-time trajectory for $c^{i}$ via the function data set BestFeasibleObjective().

Time in model calculate the mean time for all vehicles of the network:

root.timeInModel.mean()

Before experiment run:

datasetCurrentObjective.reset();

datasetBestInfeasibleObjective.reset();

datasetBestFeasibleObjective.reset();

After iteration for best object data applying logic:

if (isBestSolutionFeasible()) \{
datasetBestFeasibleObjective.update();

\}

if (!isCurrentSolutionFeasible()) \{

bestInfeasibleObjective $=\quad \min ($ bestInfeasibleObjective, getCurrentObjectiveValue()); \}

if (bestInfeasibleObjective != Double.POSITIVE_INFINITY) \{

datasetBestInfeasibleObjective.update();

\}

Table -1

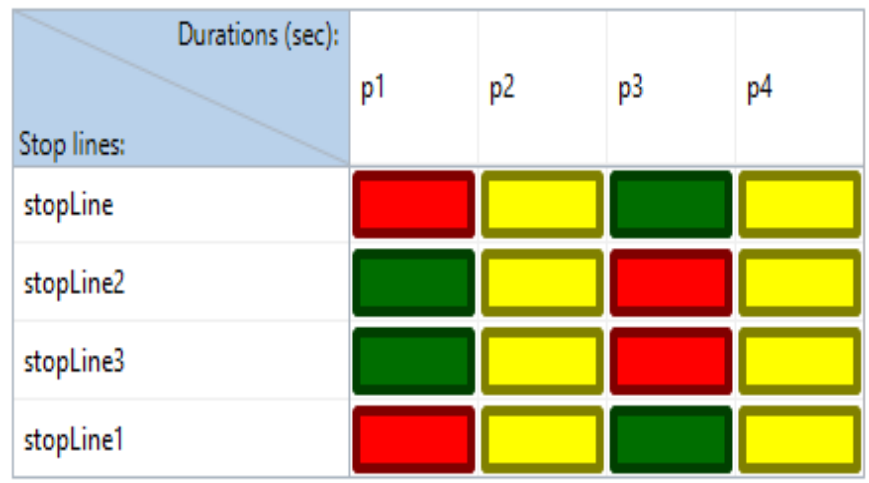

Table -1: Parameter for traffic light in mixed integer programming.

Now in algorithm MIPATS we applying traffic light parameter in discrete-time integer programming algorithm.

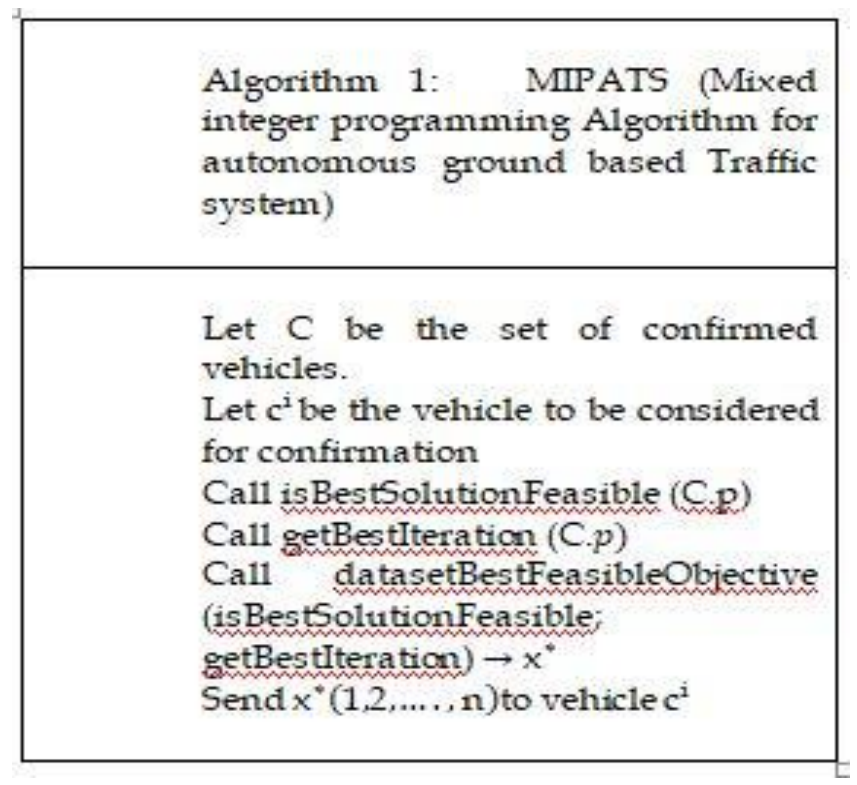




\section{Simulation}

Simulations were implemented in an ANYLOGIC [13] traffic simulator to show the performance of MIPATS. The algorithm was programmed as ALP applications. The mixedinteger programming optimization problem was resolved using ANYLOGIC optimization software.

\subsection{Simulation Setup}

In the simulations, we use a four-way crossway that has two entry lanes and two exit lanes in each way. Between the two entry lanes, the left-most lane is dedicated to left-turn vehicles and the right-turn lane can use right-turn vehicles. We can use the right two lanes by transport. The maximum allowed speed $\mathrm{v}_{\max }$ for all vehicles is $60 \mathrm{~km} / \mathrm{h}$. Vehicles enter the crossway zone with different speeds which are randomly selected in the range from $40 \%$ to $100 \%$ of $\mathrm{v}_{\max }$. All vehicles have the equivalent rectangular shape and size (5 meters long and 1:8 meters wide). We set $\mathrm{a}_{\max }=1.8 \mathrm{~m} / \mathrm{s}^{2}$ and $\mathrm{a}_{\min }=-4.2 \mathrm{~m} / \mathrm{s}^{2}$, respectively. The distance from the entrance line to the communication region of the crossway region is set as $50 \mathrm{~m}$.

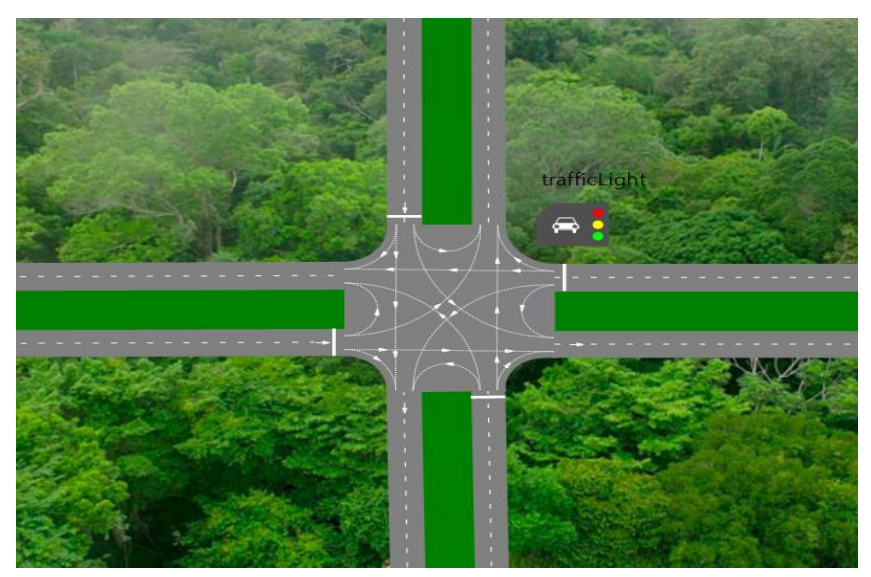

Fig 7: Routing modeled is developed for avoid collision.

In Fig-7 Vehicles are randomly selected on each entrance lane with an crossway pathway that is also randomly selected. We use several different random seeds to generate different traffic patterns and reproduce different simulations. The probabilities for a newly generated transport to take the left, through, UTurn or right pathway is $20 \%$; $40 \%$; $20 \%$, and $20 \%$ respectively. The traffic volume is represented by the average number of vehicles generated for the 10--minute simulation. We use five different traffic volumes $\{275 ; 142 ; 242 ; 267$; $237\}$. For each volume, we used simulations of four different traffic patterns to get the average results as the final result for each traffic volume. Simulations were run using $5 \mathrm{~s}$ as a time step and a simulation was terminated when the simulated time reaches 10 minutes.

We use the minimum and maximum individual type of time of each parameter for the traffic light according to table-2. All simulations were run on a 64-bit Windows computer and its processor included 4 GB RAM with Intel(R) Core(TM) i35010U CPU@2.10 GHz.

Table -2

\section{Parameters:}

\begin{tabular}{|l|l|l|l|l|}
\hline \multirow{2}{*}{ Parameter } & \multirow{4}{|l|}{ Value } \\
\cline { 3 - 5 } & Type & Min & Max & Step \\
\hline p1 & discrete & 10 & 40 & 5 \\
p2 & discrete & 4 & 20 & 5 \\
p3 & discrete & 10 & 40 & 5 \\
p4 & discrete & 4 & 20 & 5 \\
\hline
\end{tabular}

Table -2: Traffic light duration and time step according parameter

\section{CollisionFreeTrafficSystemWithMIPATS : Optimization}

\begin{tabular}{lr|r} 
& Current & Best \\
\hline Herations completed: & 4 & 6 \\
\hline Objective: $\downarrow$ & 63.944 & 39.11 \\
\hline Parameters & & Copy best \\
\hline p1 & 40 & 40 \\
\hline p2 & 19 & 4 \\
\hline p3 & 35 & 15 \\
\hline p4 & 9 & 9 \\
\hline
\end{tabular}

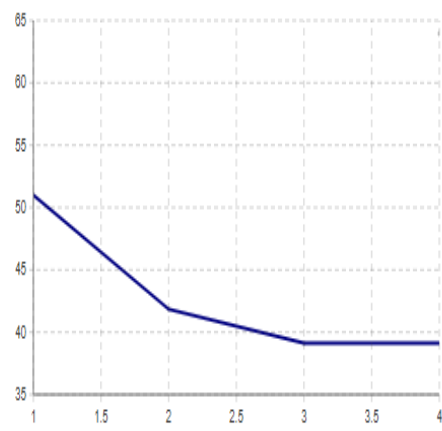

Curent Bestineasible Bestiteasible

Fig 8: Crossed vehicles' performance of the optimized traffic light with stopped time.

\subsection{Simulation Results}

For the traffic light algorithm, we determine the optimal signal cycle for different traffic volumes using the exponential cycle length mode $\mathrm{C}_{0}=1.5 \mathrm{Le}^{1.8 \mathrm{Y}}$ from [10]. In the model, $\mathrm{L}$ represents the total lost time within the cycle. The lost time for each phase is considered to be 4 seconds [11]. Since there are two phases in a cycle, the value of $L$ is set to 8 in simulations. $\mathrm{Y}$ is the sum of critical phase flow ratios. The flow ratio of a lane is obtained by dividing the demand by capacity while the critical phase flow ratio is the maximum flow ratio within the equivalent phase. The yellow light duration of each phase is 5 seconds. 
For each transport, the trip time is defined as the time it takes from the moment of entry into the communication region to the moment it exits the crossway region. The average trip time is the average of trip times of all vehicles that cross the crossway completely during the simulation. We also calculated the stopping rate, defined as the percentage of vehicles that experienced at least one-time step of stop when crossing the crossway between all crossed vehicles. If all crossed vehicles have the similar trip times, then $\mathrm{f}$ is closer to 1. If we let $\eta$ be the trip time of vehiclec ${ }^{\mathrm{i}}$, which crossed the crossway in a simulation run and $\mathrm{N}$ be the number of crossed vehicles, then we can compute the meantime as follows:

$$
\mathrm{f}\left(\vec{\eta}=\left[\eta_{1}, \eta_{2}, \ldots \ldots \ldots \eta_{N}\right]\right)=\frac{\sum_{i=1}^{\mathrm{i}=\mathrm{N}} \eta_{\mathrm{i}}}{\mathrm{N}}
$$

\begin{tabular}{|c|c|c|}
\hline \multicolumn{3}{|c|}{ timelnModel } \\
\hline count & 275 & \\
\hline Mean & 39.11 & \\
\hline Min & 4.305 & \\
\hline $\operatorname{Max}$ & 116.012 & \\
\hline Deviat & on & 21.185 \\
\hline Mean c & nfidence & 2.504 \\
\hline Sum & $10,755.2$ & \\
\hline
\end{tabular}

Fig 9: Mean time for a transport to pass the road network area.

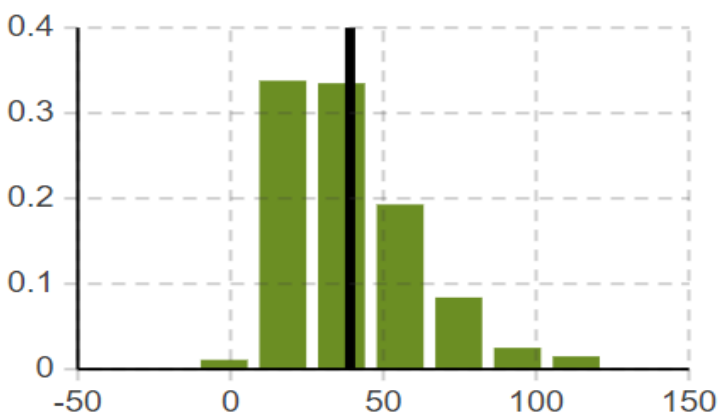

Time In Model 39.11

Fig 10: Histogram data for mean time calculate

The performance comparisons between algorithms are shown in Figure 8. The result shows that MIPATS performs significantly better than other general traffic light systems. In Fig 9, one can see that the average trip time of MIPATS remains nearly constant while those of other algorithms increase as the traffic volume increases. Also, we can see that most vehicles cross an crossway without making a complete stop under MIPATS. Even in the highest traffic volume case when more than $60 \%$ of the crossed vehicles experienced a stop under the general process, MIPATS maintains less than $21 \%$ stopped time for the equivalent situation. Fig- 8 shows the histogram data of Fig-9. Thus, the results show that the ability of MIPATS to handle the crossway crossing traffic is much larger than others. Table 3 shows The Optimized Traffic Light, Mean Value according MIPATS.

Table -3

\begin{tabular}{|c|c|c|c|c|c|}
\hline $\begin{array}{c}\text { Traffic } \\
\text { Volume } \\
\text { (Number } \\
\text { of vehicles } \\
\text { per } 10 \\
\text { minutes) }\end{array}$ & 275 & 142 & 242 & 267 & 237 \\
\hline MIPATS & 39.11 & 39.789 & 39.776 & 39.418 & 39.634 \\
\hline
\end{tabular}

Table -3: The Optimized Traffic Light, Mean Value according MIPATS

\section{CONCLUSION}

In this thesis we used the Mixed Integer Programming (MIP) to create trajectories for vehicles to follow when crossing an crossway. In formulating the optimization problem, constraints for collision avoidance and road pathway are carefully designed to ensure the protection of crossway crossing vehicles. The simulation results show that the suggested MIPATS (Mixed integer programming Algorithm for self-directed ground-based Traffic system) achieves reasonably higher throughput as well as a justification than general crossway control strategies . Currently, we are improving MIPATS to make it applicable in practice by incorporating more realistic V2I wireless communication characteristics into the framework through an optimized traffic light system. Also, we planned to develop MIPATS on an crossway network to optimize the traffic throughput performance on a larger scale. In the future, it will be possible to reduce the meantime of a traffic network from the above meantime. We worked on this suggested algorithm MIPATS by optimizing other constraints of a car agent and traffic system such as speed, acceleration, deceleration, car length, median width, car density, and probability of car in the pathway , etc.

\section{REFERENCES}

[1] F. Zhu and S. V. Ukkusuri, "A linear programming formulation for autonomous intersection control within a dynamic traffic assignment and connected transport environment," Transportation Research Part C: Emerging Technologies, vol. 55, pp. 363-378, 2015.

[2] K.-D. Kim and P. R. Kumar, "An mpc-based formulation to provable system-wide safety and liveness of autonomous ground traffic," IEEE Trans. Autom. Control, vol. 59, no. 12, pp. 3341-3356, 2014.

[3] A. A. Malikopoulos, C. G. Cassandras, and Y. J. Zhang, "A decentralized optimal control framework for 
connected and automated vehicles at urban intersections," arXiv preprint arXiv:1602.03786, 2016.

[4] K. Dresner and P. Stone, "A multiagent formulation to autonomous intersection management," JAIR, vol. 31, pp. 591-656, 2008.

[5] P. Dai, K. Liu, Q. Zhuge, E. H.-M. Sha, V. C. S. Lee, and S. H. Son, "Quality-of-experience-oriented autonomous intersection control in vehicular networks," IEEE Trans. Intell. Transp. Syst., vol. 17, no. 7, pp. 1956-1967, 2016.

[6] M. A. S. Kamal, J.-i. Imura, T. Hayakawa, A. Ohata, and K. Aihara, "A vehicle-intersection coordination scheme for smooth flows of traffic without using traffic lights," IEEE Trans. Intell. Transp. Syst., vol. 16, no. 3, pp. 1136-1147, 2015

[7] Q. Lu and K.-D. Kim, "Intelligent intersection management of autonomous traffic using discrete-time occupancies trajectory," Journal of Traffic and Logistics Engineering Vol, vol. 4, no. 1, pp. 1-6, 2016.

[8] Q. Lu and K.-D. Kim "Autonomous and connected intersection crossing traffic management using discretetime occupancies trajectory," arXiv preprint arXiv:1705.05231, 2017.

[9] Q. Lu and K.-D. Kim, "A genetic algorithm formulation for expedited crossing of emergency vehicles in connected and autonomous intersection traffic," Journal of Advanced Transportation, 2017.

[10] D. Cheng, Z. Z. Tian, and C. J. Messer, "Development of an improved cycle length model over the highway capacity manual 2000 quick estimation method," Journal of transportation engineering, vol. 131, no. 12, pp. 890897, 2005.

[11] T. R. Board, Highway Capacity Manual, National Academy of Sciences, Transportation Research Board, Washington, DC, 2000.

[12] R. Jain, D.-M. Chiu, and W. R. Hawe, A quantitative measure of fairness and discrimination for resource allocation in shared computer system. Eastern Research Laboratory, Digital Equipment Corporation Hudson, MA, 1984, vol. 38 .

[13] I. ANYLOGIC, “ANYLOGIC reference manual," 2019. [Online]. Available: https://www.anylogic.com/ 